\title{
Risk Factors for Human Brucellosis in Northern Tanzania
}

\begin{abstract}
Shama Cash-Goldwasser, ${ }^{1,2 \star}$ Michael J. Maze, ${ }^{2,3}$ Matthew P. Rubach, ${ }^{2,4}$ Holly M. Biggs, ${ }^{4}$ Robyn A. Stoddard, ${ }^{5}$ Katrina J. Sharples, ${ }^{6,7}$ Jo E. B. Halliday, ${ }^{8}$ Sarah Cleaveland, ${ }^{8}$ Michael C. Shand, ${ }^{8}$ Blandina T. Mmbaga, ${ }^{1,2,9}$ Charles Muiruri, ${ }^{1}$ Wilbrod Saganda, ${ }^{10}$ Bingileki F. Lwezaula, ${ }^{10}$ Rudovick R. Kazwala, ${ }^{11}$ Venance P. Maro, ${ }^{2,9}$ and John A. Crump ${ }^{1,3,4,9}$

${ }^{1}$ Duke Global Health Institute, Duke University, Durham, North Carolina; ${ }^{2}$ Kilimanjaro Christian Medical Centre, Moshi, Tanzania; ${ }^{3}$ Centre for International Health, University of Otago, Dunedin, New Zealand; ${ }^{4}$ Division of Infectious Diseases, Duke University Medical Center, Durham, North Carolina; ${ }^{5}$ Centers for Disease Control and Prevention, Bacterial Special Pathogens Branch, Atlanta, Georgia; ${ }^{6}$ Department of Mathematics and Statistics, University of Otago, Dunedin, New Zealand; ${ }^{7}$ Department of Medicine, University of Otago, Dunedin, New Zealand; ${ }^{8}$ Boyd Orr Centre for Population and Ecosystem Health, Institute of Biodiversity, Animal Health and Comparative Medicine, University of Glasgow, Glasgow, United Kingdom; ${ }^{9}$ Kilimanjaro Christian Medical University College, Moshi, Tanzania; ${ }^{10}$ Mawenzi Regional Referral Hospital, Moshi,Tanzania;

${ }^{11}$ Department of Veterinary Medicine and Public Health, Sokoine University of Agriculture, Morogoro, Tanzania
\end{abstract}

\begin{abstract}
Little is known about the epidemiology of human brucellosis in sub-Saharan Africa. This hampers prevention and control efforts at the individual and population levels. To evaluate risk factors for brucellosis in northern Tanzania, we conducted a study of patients presenting with fever to two hospitals in Moshi, Tanzania. Serum taken at enrollment and at 4-6 week follow-up was tested by Brucella microagglutination test. Among participants with a clinically compatible illness, confirmed brucellosis cases were defined as having a $\geq 4$-fold rise in agglutination titer between paired sera or a blood culture positive for Brucella spp., and probable brucellosis cases were defined as having a single reciprocal titer $\geq 160$. Controls had reciprocal titers $<20$ in paired sera. We collected demographic and clinical information and administered a risk factor questionnaire. Of 562 participants in the analysis, $50(8.9 \%)$ had confirmed or probable brucellosis. Multivariable analysis showed that risk factors for brucellosis included assisting goat or sheep births (Odds ratio $[\mathrm{OR}] 5.9,95 \%$ confidence interval $[\mathrm{Cl}] 1.4,24.6)$ and having contact with cattle (OR 1.2, 95\% $\mathrm{Cl} 1.0,1.4)$. Consuming boiled or pasteurized dairy products was protective against brucellosis (OR $0.12,95 \% \mathrm{Cl} 0.02,0.93$ ). No participants received a clinical diagnosis of brucellosis from their healthcare providers. The under-recognition of brucellosis by healthcare workers could be addressed with clinician education and better access to brucellosis diagnostic tests. Interventions focused on protecting livestock keepers, especially those who assist goat or sheep births, are needed.
\end{abstract}

\section{INTRODUCTION}

Human brucellosis is a major zoonosis worldwide. ${ }^{1,2}$ It presents as an acute febrile illness ${ }^{3,4}$ and sometimes progresses to chronic debilitating disease. ${ }^{5}$ In addition to direct impacts on human health, brucellosis is associated with reproductive failure in domestic animals, resulting in economic losses for communities that rely on livestock for their livelihoods. ${ }^{6,7}$

Prevention of human brucellosis hinges on disease control in the livestock reservoir. ${ }^{1}$ Livestock vaccination and test and slaughter programs have been used in some countries to achieve elimination of both livestock and human brucellosis. ${ }^{1,8,9}$ However, such approaches have rarely been used in subSaharan Africa because implementation resources are scarce and the case for investment has not been made., ${ }^{2,7}$ In addition, the Brucella and livestock species that drive brucellosis epidemiology in sub-Saharan Africa remain unknown. ${ }^{9}$ While Brucella melitensis typically infects goats and sheep, and Brucella abortus typically infects cattle, cross-species infections have complicated control efforts in some places. ${ }^{6}$

Brucella localizes to the reproductive tract and mammary glands of livestock and may be present in the blood, reproductive tract secretions, and milk. ${ }^{10}$ Humans may acquire the infection through direct contact, foodborne transmission, or airborne transmission if an infectious source is aerosolized. ${ }^{11}$ Risk factors for human brucellosis vary by context because of different animal reservoirs and behavioral practices. For example, brucellosis has been associated with consumption of camel milk in Israel, ${ }^{12}$ with slaughtering pigs in

${ }^{*}$ Address correspondence to Shama Cash-Goldwasser, Fogarty Global Health Fellow, Duke Global Health Institute, Duke University, Box 90519, Durham, NC 27710. E-mail: shamacg@gmail.com the United States, ${ }^{13}$ and with exposure to livestock placentas in Chad. ${ }^{14}$

Brucellosis transmission to humans can be interrupted through behavior change, provision of personal protective equipment that limits human exposure to infectious sources, and food safety interventions that target meat or milk production. ${ }^{1}$ Such interventions rely on knowledge of the burden of brucellosis and specific local risk factors. A study on the etiology of febrile illness among hospitalized patients in northern Tanzania showed that 16 (3.5\%) of 453 participants had confirmed acute brucellosis. ${ }^{15}$ The prevalence of antibodies against Brucella species among abattoir workers in northern Tanzania was $8 \% .{ }^{16}$ Human brucellosis in Tanzania has been associated with assisting livestock births, ${ }^{17}$ and Brucella antibody seropositivity has been associated with slaughtering livestock, ${ }^{16}$ and with milking, herding, or assisting cattle births. ${ }^{18}$ These data imply that brucellosis is endemic in northern Tanzania and that exposure may occur through a range of livestock-oriented activities.

To identify locally appropriate prevention measures, more data on risk factors for active human disease, rather than for Brucella antibody seropositivity, are needed. In addition, it is necessary to determine which specific activities involving which livestock species are associated with greatest risk. To investigate risk factors for human brucellosis in northern Tanzania, we conducted a prospective cohort study of febrile patients using a rigorous brucellosis case definition and a detailed risk factor questionnaire.

\section{MATERIALS AND METHODS}

Study setting. We conducted our study at two hospitals in Moshi, Tanzania. Mawenzi Regional Referral Hospital (MRRH) 
is a 210-bed regional hospital serving the Kilimanjaro Region. Kilimanjaro Christian Medical Center (KCMC) is a 450-bed consultant referral hospital that serves a large catchment area, including the Kilimanjaro Region and other regions, in northern Tanzania. Moshi (population $>180,000$ ) is the administrative center of Kilimanjaro Region (population $>1.6$ million) ${ }^{19}$ and is situated at an elevation of approximately 890 meters above sea level. The climate is tropical, with rainy seasons from October through December and from March through May. The Kilimanjaro Region is predominately rural. ${ }^{19}$ Agriculture in northern Tanzania is characterized by pastoralism and by a mix of smallholder systems involving mixed crop and livestock farming. ${ }^{20}$

Study procedures and participants. We enrolled pediatric and adult patients presenting to $\mathrm{MRRH}$ and $\mathrm{KCMC}$ from February 2012 through May 2014. On weekdays, we screened all patients admitted within the past 24 hours to the adult and pediatric medical wards at $\mathrm{MRRH}$ and to the adult medical ward at KCMC, as well as patients presenting to the outpatient department at MRRH. We enrolled consecutive eligible inpatients and every second eligible outpatient. Inpatients and outpatients were eligible to participate if they had an axillary temperature of $>37.5^{\circ} \mathrm{C}$, or a tympanic, oral, or rectal temperature of $\geq 38.0^{\circ} \mathrm{C}$ at presentation. Inpatients were also eligible if they reported a history of fever within the past 72 hours. After obtaining informed consent, a trained study team member collected demographic and clinical information and administered a standardized risk factor questionnaire. The risk factor questionnaire included questions on dietary practices and daily activities performed within the past month, with a focus on agricultural and animal-related activities. Blood was drawn for aerobic blood culture, examination for blood parasites, and acute serum archiving. Healthcare workers who were not part of the study team delivered outpatient and inpatient care according to local hospital standards. Clinical diagnoses at patient discharge were recorded. We immediately communicated the results of critical laboratory tests, including blood parasite smears and blood cultures to responsible medical personnel. The Brucella serology results were provided once available. Participants were asked to return 4-6 weeks after enrollment for collection of a convalescent serum sample. Study personnel visited participant households to collect Global Positioning System (GPS) coordinates of the households.

Laboratory methods and case definitions. Blood cultures were performed using BacT/ALERT pediatric fastidious bottles for children or standard aerobic bottles for adults, which were loaded into the BacT/ALERT 3D Microbial Detection system (BioMerieux Inc., Durham, NC) and incubated for 5 days. Standard methods were used for identifying bloodstream isolates. ${ }^{3,21}$ Thick and thin blood smears were examined for blood parasites by certified laboratory technologists. We sent acute and convalescent serum samples to the US Centers for Disease Control and Prevention (CDC) for analysis using the Brucella microagglutination test (BMAT). Standardized $B$. abortus strain 1119-3 killed antigen (National Veterinary Services Laboratory, Ames, IA) was used for BMAT at a 1:25 working dilution. Results were read on a Scienceware Plate Reader (Bel-Art Products, Wayne, NJ). Minor modifications were made to the CDC's standard BMAT, including the use of U-bottom plates, incubation at $26^{\circ} \mathrm{C}$, and discontinued use of safranin. ${ }^{22}$
We defined brucellosis as a clinically compatible illness plus laboratory evidence of infection. For confirmed cases, laboratory evidence was either $\mathrm{a} \geq 4$-fold rise in Brucella antibody titer between acute and convalescent serum samples or a blood culture positive for Brucella spp. For probable cases, laboratory evidence was a single reciprocal titer $\geq 160 .{ }^{23}$ In the analysis, we pooled participants who met criteria for confirmed or probable brucellosis and classified them as brucellosis cases. We classified participants with reciprocal titers $<20$ in both acute and convalescent serum samples as controls. Once BMAT results were available, the study team attempted to trace participants who met our brucellosis case definition so that untreated brucellosis could be managed.

Geospatial data and definitions. Population data were obtained from the National Bureau of Statistics, Tanzania. We grouped participants with household GPS data according to population density: urban zones had a population density of $\geq 1,000$ inhabitants $/ \mathrm{km}^{2}$, peri-urban zones had a population density of $\geq 300$ inhabitants $/ \mathrm{km}^{2}$ and were $\leq 15 \mathrm{~km}$ distance from urban zones, and rural zones had a population density of $<300$ inhabitants $/ \mathrm{km}^{2}$ or were $>15 \mathrm{~km}$ distance from urban zones. Population density was calculated from the 2012 Tanzania Population and Housing Census. ${ }^{19}$

Statistical analysis. Data were entered using the Cardiff Teleform system (Cardiff Inc., Vista, CA) into an Access database (Microsoft Corporation, Redmond, WA). Geospatial data were managed using QGIS, version 2.12.0 (Free Software Foundation, Boston, MA). Analyses were performed using STATA, version 13.1 (STATA-Corp, College Station, TX). The spatial scan statistic was performed using a Bernoulli model to assess for evidence of spatial clustering among cases using SatScan version 9.0 (www.satscan.org). We derived a socioeconomic status scale using principal components analysis. $^{24}$

Because of the high ratio of independent variables to cases and resultant instability of our multivariable models, we combined independent variables to reduce the number in the

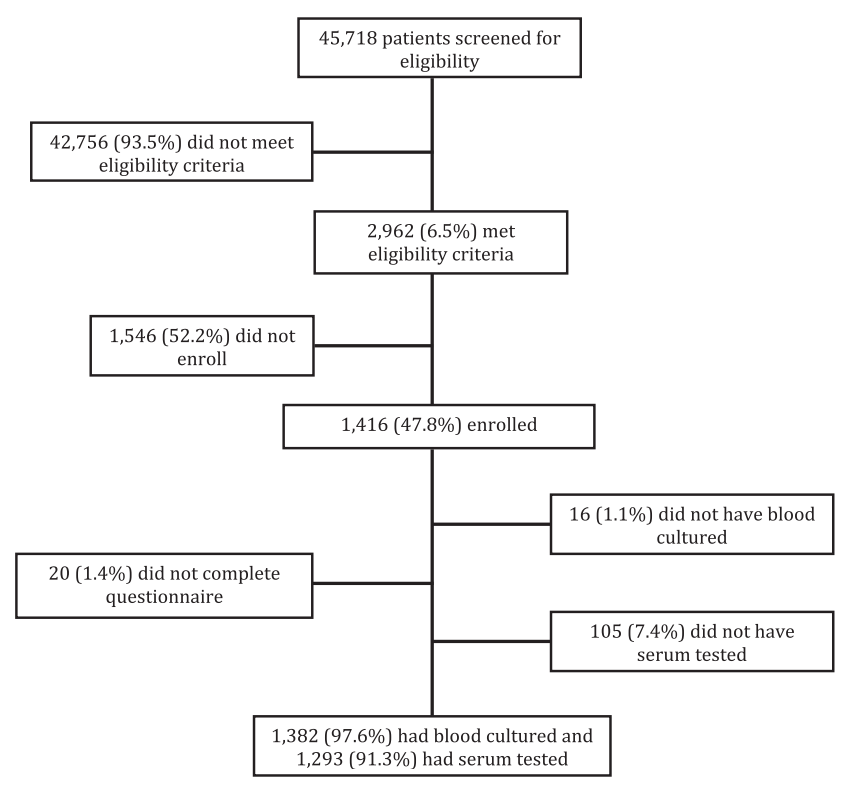

FIGURE 1. Study flow diagram for patients seeking care at Kilimanjaro Christian Medical Center and Mawenzi Regional Hospital in Moshi, Tanzania, 2012-2014. 
analysis. We used a method of variable aggregation that also allowed us to quantitatively measure participant exposure to potential risk factors for brucellosis. We already had single variables that represented participant dairy exposure and participant livestock birthing exposure, so we created aggregated variables or exposure scales, to measure participant livestock blood exposure and participant livestock contact. We used an analytic hierarchy process to develop these exposure scales. ${ }^{25}$ First, we identified relevant behaviors and living conditions from the risk factor questionnaire to be included in each scale. We then identified locally experienced subject matter experts, including epidemiologists, livestock workers, physicians, and veterinarians. We asked experts to rank every behavior in a particular scale against all other behaviors in that scale, in terms of the likelihood of livestock blood exposure or the likelihood of livestock contact, using a nine-point bidirectional scale. We then combined the rankings to obtain each variable's weight. ${ }^{26}$ To derive the consensus weight of each behavior, we calculated the geometric mean of the experts' weights. We included only the weights assigned by experts who provided internally consistent answers, defined as achieving a consistency ratio $<0.2$. $^{26}$ To aid interpretation of exposure scores, we scaled the weights so that the minimum possible score on each scale was 0 and the maximum possible score was 20. For both the livestock blood exposure scale and the livestock contact scale, we produced versions for cattle, goats, pigs, and sheep in aggregate, for cattle alone, for pigs alone, and for goats and sheep together. Finally, we derived a score for each participant on each exposure scale depending on the reported frequency of relevant behaviors in the questionnaire. For example, if someone performed none of the activities in the cattle blood exposure scale, they would score " 0 " on that scale, and if they performed every activity in the sheep blood exposure scale, they would score "20" on that scale.

Participants who did not meet the definition of either a brucellosis case or a control were excluded from the analyses. Univariable logistic regression was performed to explore associations between potential risk factors and risk of brucellosis. The models grouped goats and sheep together. Odds ratios (ORs) and 95\% confidence intervals $(\mathrm{Cls})$ were reported when appropriate. We built a multivariable model to examine associations between multiple risk factors and odds of brucellosis. Decisions to include variables in the model were based on known or suspected associations with brucellosis, and both individual behaviors and exposure scales were included. The forms of the relationships between the exposure scales and brucellosis risk were determined using fractional polynomial models. Backward selection guided by the Akaike information criterion was used to arrive at a final model. All $P$ values were two sided, and $P<0.05$ was considered statistically significant.

Research ethics. Written informed consent was obtained from all adult participants and from the parents or legal guardians of minors. This study was approved by the KCMC

TABLE 1

Demographic and clinical characteristics of participants with and without brucellosis, northern Tanzania, 2012-2014

\begin{tabular}{|c|c|c|c|c|c|c|}
\hline & \multicolumn{2}{|c|}{ With brucellosis $(N=50)^{*}$} & \multicolumn{2}{|c|}{ Without brucellosis $(N=512)^{*}$} & \multirow[b]{2}{*}{ OR $(95 \% \mathrm{Cl})$} & \multirow[b]{2}{*}{$P$ value } \\
\hline & $n$ & (\%) & $n$ & (\%) & & \\
\hline \multicolumn{7}{|l|}{ Demographics } \\
\hline Age, median (range) years & $30.57(0.57,77.18)$ & $\mathrm{n} / \mathrm{a}$ & $20.55(0.22,93.5)$ & $\mathrm{n} / \mathrm{a}$ & $1.6(1.2,2.1)$ & 0.001 \\
\hline Female sex & 33 & $(66.0)$ & 273 & $(53.3)$ & $1.7(0.89,3.3)$ & 0.115 \\
\hline \multicolumn{7}{|l|}{ Occupation† } \\
\hline Butcher & 0 & $(0.0)$ & 3 & $(0.6)$ & - & - \\
\hline Farmer & 15 & $(30.0)$ & 90 & (17.6) & $2.0(1.0,4.0)$ & 0.060 \\
\hline Livestock attendant & 1 & $(2.0)$ & 7 & (1.4) & $1.8(0.53,5.2)$ & 0.352 \\
\hline Vet & 0 & $(0.0)$ & 1 & $(0.2)$ & - & - \\
\hline Other $\ddagger$ & 34 & $(68.0)$ & 410 & $(80.1)$ & $0.53(0.27,1.1)$ & 0.077 \\
\hline Pastoralist tribe§ & 0 & $(0.0)$ & 8 & $(3.9)$ & - & - \\
\hline \multicolumn{7}{|l|}{ Population density category } \\
\hline Rural & 11 & (23.9) & 109 & $(25.5)$ & $\mathrm{n} / \mathrm{a}$ & $\mathrm{n} / \mathrm{a}$ \\
\hline Peri-urban & 10 & (21.7) & 87 & (20.3) & $1.1(0.46,2.8)$ & 0.777 \\
\hline Urban & 25 & (54.3) & 232 & $(54.2)$ & $1.0(0.51,2.2)$ & 0.863 \\
\hline Residence in Moshi Urban District & 28 & (56.0) & 245 & (47.9) & $0.75(0.40,1.4)$ & 0.419 \\
\hline \multicolumn{7}{|l|}{ Socioeconomic status } \\
\hline Lowest 25th percentile & 18 & $(36.0)$ & 119 & (23.2) & $\mathrm{n} / \mathrm{a}$ & $\mathrm{n} / \mathrm{a}$ \\
\hline Middle 50th percentile & 19 & $(38.0)$ & 264 & (51.6) & $0.48(0.24,0.94)$ & 0.032 \\
\hline Highest 75th percentile & 13 & (26.0) & 129 & (25.2) & $0.67(0.31,1.4)$ & 0.292 \\
\hline \multicolumn{7}{|l|}{ Clinical history $†$} \\
\hline Gastrointestinal symptoms & 47 & $(94.0)$ & 459 & (89.6) & $1.8(0.55,9.4)$ & 0.480 \\
\hline Musculoskeletal symptoms & 39 & (78.0) & 297 & (58.0) & $2.6(1.3,5.7)$ & 0.007 \\
\hline Neurologic symptoms & 44 & $(88.0)$ & 330 & (64.5) & $4.03(1.7,11.8)$ & 0.001 \\
\hline Respiratory symptoms & 26 & (52.0) & 338 & $(66.0)$ & $0.56(0.30,1.0)$ & 0.071 \\
\hline \multicolumn{7}{|l|}{ Diagnosis $†$} \\
\hline Clinical diagnosis brucellosis & 0 & $(0.0)$ & 0 & $(0.0)$ & - & - \\
\hline Clinical diagnosis malaria & 13 & $(26.0)$ & 116 & $(22.7)$ & $1.2(0.57,2.4)$ & 0.702 \\
\hline Laboratory confirmed malaria & 2 & $(4.0)$ & 11 & $(2.2)$ & $1.9(0.20,9.0)$ & 0.659 \\
\hline
\end{tabular}

$\mathrm{Cl}=$ confidence interval; $\mathrm{OR}=$ odds ratio.

* Data not available for all participants for all variables; \% reflects the accurate denominator.

† Categories not mutually exclusive.

$\ddagger$ Other - artisan, driver, guard, healthcare worker, manual laborer, miner, office worker, police, student, teacher, unemployed.

§astoralist tribe - Barabaig or Maasai.

TReference category in regression analysis. 
Research Ethics Committee, the Tanzania National Institute for Medical Research National Research Ethics Coordinating Committee, an Institutional Review Board of Duke University Health System, the University of Otago Human Ethics Committee (Health), and the US CDC.

\section{RESULTS}

Participant enrollment and characteristics. Participant enrollment is summarized in Figure 1. Of 1,382 participants who had blood cultured, 63 (4.6\%) grew pathogenic species but none grew Brucella spp. Of 1,293 participants who had serum tested for Brucella antibodies, 731 (56.5\%) were excluded from the analysis because they did not meet brucellosis case or control definitions, $292(22.3 \%)$ had an antibody titer $\geq 20$ and $<160$ in at least one serum sample, 439 (33.5\%) had a titer $<20$ in one serum sample but were missing a second sample, and 562 (43.5\%) were included in the analysis. Of the 562 included in the analysis, 50 (8.9\%) had brucellosis; 39 (6.9\%) were confirmed cases and 11 (2.0\%) were probable cases.

Participant demographic and clinical characteristics, and associated univariable regression results, are presented in Table 1. No participant received a clinical diagnosis of brucellosis. One hundred and twenty-nine (23.0\%) of 562 participants received a clinical diagnosis of malaria, whereas $13(2.3 \%)$ of 556 participants with available blood microscopy results had laboratory-confirmed malaria. Most

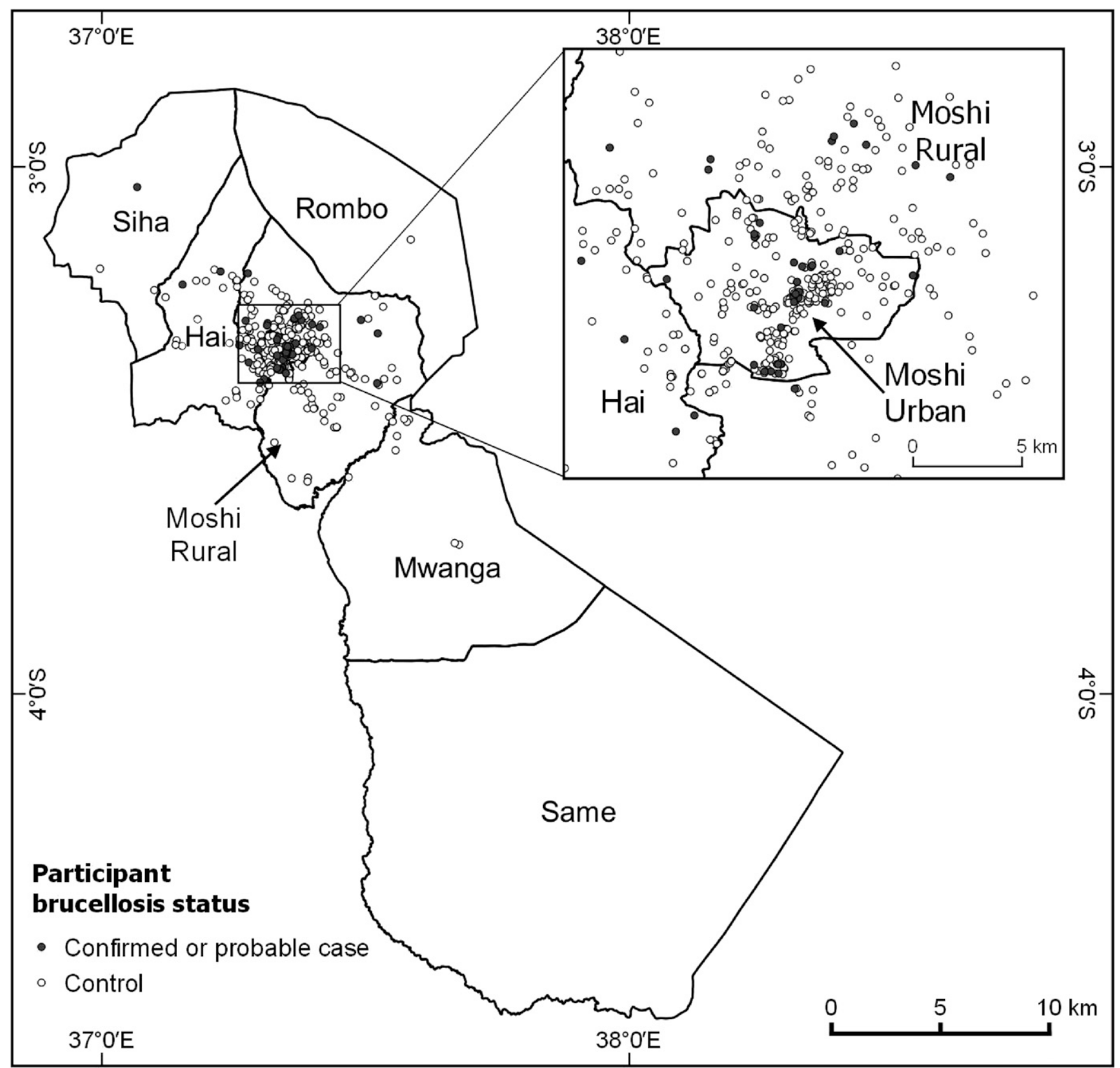

FIGURE 2. Location by district of participants with and without brucellosis, Kilimanjaro Region, northern Tanzania, 2012-2014. 
participants had multiple presenting complaints in addition to fever.

Three hundred and ninety-four $(70.1 \%)$ participants were over the age of 5 years. Older age was associated with brucellosis (OR 1.5 per year increase in age, $\mathrm{Cl} 1.1,2.0)$. Eight $(1.4 \%)$ were from a pastoralist tribe. One hundred and five (18.7\%) participants were farmers, and this was the most commonly reported livestock-related profession. No livestockrelated occupation was associated with brucellosis. For 273 (48.6\%) participants, the self-reported district of residence was Moshi Urban District. Geospatial coordinates were available for 474 (84.3\%) participant households. Of those, 120 (25.3\%) were in a rural zone, 97 (20.5\%) were in a peri-urban zone, and 257 (54.2\%) were in an urban zone. No specific zone was associated with brucellosis. There was no evidence of clustering in the spatial distribution of cases. Brucellosis cases and controls are mapped in Figure 2.

Exposure scales. To derive the livestock contact scale and the livestock blood exposure scale, we used the weights assigned to behaviors by eight and six internally consistent experts, respectively. Table 2 shows the variable weights that comprised each exposure scale. In the livestock contact scale, seeing livestock around the house had the lowest weight (0.65), whereas slaughtering livestock had the highest weight (4.19). In the livestock blood exposure scale, assisting livestock abortions had the lowest weight (0.87), whereas consuming raw livestock blood had the highest weight (8.52).

Univariable analysis. Univariable regression results for behaviors and exposure scores on brucellosis are presented in Table 3. A number of livestock-related activities were associated with brucellosis, including assisting cattle births (OR $10.3,95 \% \mathrm{Cl} 0.13,820.1$ ), assisting goat or sheep births (OR $7.2,95 \% \mathrm{Cl} 1.5,32.0)$, cleaning cattle waste (OR 4.1, 95\% Cl $1.6,9.8)$, cleaning pig waste (OR 5.5, 95\% Cl 1.4, 18.8), feeding livestock (OR 2.8, 95\% Cl 1.4, 5.5), and consuming raw blood of livestock (OR 2.7, 95\% Cl 1.1, 6.3). Contact with livestock was associated with brucellosis (OR 1.2 per point increase in score, $95 \% \mathrm{Cl} 1.0,1.3)$, as was contact with each individual livestock species. Contact with livestock blood was associated with brucellosis (OR 1.1 per point increase in score, 95\% $\mathrm{Cl} 1.0,1.2)$, but contact with the blood of each individual livestock species was not.

Multivariable analysis. A multivariable model was constructed to explore independent associations between brucellosis and contact with each livestock species, contact with the blood of each livestock species, assisting births of each livestock species, consumption of raw dairy or dairy products, and consumption of boiled or pasteurized dairy and dairy products. Results are shown in Table 4. Of 562 participants, two $(0.36 \%)$ had missing values for variables in the multivariable model and were dropped from the multivariable analysis. We controlled for age and for reported district of residence. Brucellosis was associated with assisting goat or sheep births (OR 5.9, 95\% Cl 1.4, 25.2) and with cattle contact (OR 1.2 per point increase in score, $95 \% \mathrm{Cl} 1.0,1.4)$. Consuming boiled or pasteurized dairy products was identified as a protective factor (OR 0.12, 95\% Cl 0.02, 0.91). While consumption of raw dairy products was not associated with brucellosis (OR 0.89 , $\mathrm{Cl} 0.43,1.9)$, model fit was better with inclusion of this variable.

\section{DISCUSSION}

Our results point to multiple potential transmission pathways involving several livestock species in the epidemiology of human brucellosis in northern Tanzania. We showed that assisting goat or sheep births and contact with cattle were risk factors for brucellosis. We found that consuming boiled or pasteurized dairy products was protective. We also confirmed that brucellosis remains underdiagnosed by healthcare workers.

The association between assisting goat or sheep births and risk for brucellosis is consistent with the localization of Brucella to the reproductive tract of livestock. Indeed, other studies from Tanzania have demonstrated a relationship between exposure to livestock reproductive tract secretions and brucellosis or Brucella antibody seropositivity. ${ }^{17,18,27}$ Our finding that the riskiest behavior was assisting goat or sheep births is consistent with an analysis of human and livestock serologic data from northern Tanzania, which showed that human Brucella antibody seropositivity was more likely associated with goat and sheep contact than with cattle contact. ${ }^{28}$

We found evidence of an association between brucellosis and cattle contact. This is in agreement with other studies from sub-Saharan Africa that have implicated cattle as an important reservoir, ${ }^{17,29,30}$ and it indicates a potential role for cattle in the epidemiology of brucellosis in northern Tanzania. Our findings that brucellosis is associated both with cattle contact and with assisting goat or sheep births may suggest that both $B$. abortus and $B$. melitensis are circulating in northern Tanzania, or that a single Brucella species is infecting multiple livestock species.

TABLE 2

Variables included in the exposure scales for participants with and without brucellosis, northern Tanzania, 2012-2014

\begin{tabular}{lccc}
\hline \multicolumn{1}{c}{ Livestock contact scale } & & \multicolumn{2}{c}{ Livestock blood exposure scale } \\
\cline { 3 - 4 } \multicolumn{1}{c}{ Variable } & Weight & & Variable \\
See livestock around house & 0.65 & Assist livestock abortions & Weight \\
Keep livestock around house & 0.84 & Touch livestock carcass & 0.87 \\
Assist livestock abortions & 1.15 & Veterinarian & 1.25 \\
Feed livestock & 1.15 & Assist livestock births & 1.53 \\
Clean livestock waste & 1.18 & Slaughter livestock & 1.82 \\
Keep livestock inside house & 1.56 & Consume raw livestock blood & 6.02 \\
Livestock attendant & 1.57 & - & - \\
Assist livestock births & 1.65 & - & - \\
Veterinarian & 2.18 & - & - \\
Milk livestock & 2.35 & - & - \\
Slaughter livestock & 4.19 & & - \\
\hline
\end{tabular}


TABLE 3

Univariable analysis of behaviors and exposures for participants with and without brucellosis, northern Tanzania, 2012-2014

\begin{tabular}{|c|c|c|c|c|c|c|}
\hline & \multicolumn{2}{|c|}{ With brucellosis $(N=50)^{\star}$} & \multicolumn{2}{|c|}{ Without brucellosis $(N=512)^{\star}$} & \multirow[b]{2}{*}{ OR $(95 \% \mathrm{Cl})$} & \multirow[b]{2}{*}{$P$ value } \\
\hline & $n$ & (\%) & $n$ & (\%) & & \\
\hline \multicolumn{7}{|l|}{ Activities with livestock $\dagger$} \\
\hline Assist livestock abortions & 3 & $(6.0)$ & 12 & (2.3) & $2.7(0.46,10.3)$ & 0.285 \\
\hline Cattle & 0 & $(0.0)$ & 2 & $(0.4)$ & - & - \\
\hline Goats or sheep & 1 & (2.0) & 9 & (1.8) & $1.1(0.03,8.5)$ & 1.000 \\
\hline Pigs & 2 & $(4.0)$ & 1 & $(0.2)$ & $21.0(1.1,1,258.3)$ & 0.044 \\
\hline Assist livestock births & 4 & (8.0) & 7 & (1.4) & $6.2(1.3,25.6)$ & 0.023 \\
\hline Cattle & 1 & (2.0) & 1 & $(0.2)$ & $10.3(0.13,820.1)$ & 0.040 \\
\hline Goats or sheep & 4 & (8.0) & 6 & (1.2) & $7.2(1.5,32.0)$ & 0.000 \\
\hline Pigs & 0 & $(0.0)$ & 0 & $(0.0)$ & - & - \\
\hline Clean livestock waste & 9 & (18.0) & 47 & (9.2) & $2.2(0.87,4.9)$ & 0.097 \\
\hline Cattle & 9 & (18.0) & 26 & (5.1) & $4.1(1.6,9.8)$ & 0.000 \\
\hline Goats or sheep & 4 & $(8.0)$ & 30 & (5.9) & $1.4(0.34,4.2)$ & 0.545 \\
\hline Pigs & 5 & (10.0) & 10 & (2.0) & $5.5(1.4,18.8)$ & 0.001 \\
\hline Feed livestock & 16 & (32.0) & 74 & (14.5) & $2.8(1.4,5.5)$ & 0.001 \\
\hline Cattle & 14 & $(28.0)$ & 46 & $(9.0)$ & $3.9(1.8,8.1)$ & 0.000 \\
\hline Goats or sheep & 11 & (22.0) & 53 & (10.4) & $2.4(1.1,5.2)$ & 0.013 \\
\hline Pigs & 5 & (10.0) & 8 & $(1.6)$ & $1.0(1.7,25.3)$ & 0.000 \\
\hline Herd livestock & 4 & $(8.0)$ & 17 & (3.3) & $2.5(0.59,8.2)$ & 0.374 \\
\hline Cattle & 2 & (4.0) & 7 & (1.4) & $3.0(0.30,16.3)$ & 0.214 \\
\hline Goats or sheep & 4 & (8.0) & 17 & (3.3) & $2.5(0.59,8.2)$ & 0.214 \\
\hline Keep livestock around house & 15 & (30.0) & 174 & (34.0) & $0.83(0.41,1.6)$ & 0.690 \\
\hline Cattle & 12 & $(24.0)$ & 117 & (22.9) & $1.1(0.49,2.2)$ & 0.972 \\
\hline Goats or sheep & 11 & (22.0) & 138 & (27.0) & $0.76(0.34,1.6)$ & 0.566 \\
\hline Pigs & 0 & $(0.0)$ & 0 & $(0.0)$ & - & - \\
\hline Keep livestock in house & 8 & (16.0) & 45 & (8.8) & $2.0(0.75,4.6)$ & 0.170 \\
\hline Cattle & 2 & $(4.0)$ & 4 & $(0.8)$ & $5.3(0.46,37.8)$ & 0.185 \\
\hline Goats or sheep & 1 & (2.0) & 6 & (1.2) & $1.7(0.04,14.6)$ & 0.962 \\
\hline Pigs & 6 & (12.0) & 39 & (7.6) & $1.6(0.54,4.2)$ & 0.401 \\
\hline Milk livestock & 3 & $(6.0)$ & 15 & (2.9) & $2.1(0.38,7.9)$ & 0.419 \\
\hline Cattle & 3 & $(6.0)$ & 15 & (2.9) & $2.1(0.38,7.9)$ & 0.419 \\
\hline Goats or sheep & 0 & $(0.0)$ & 2 & $(0.4)$ & - & - \\
\hline Own livestock & 19 & $(38.0)$ & 195 & (38.1) & $1.0(0.52,1.9)$ & 0.991 \\
\hline Cattle & 15 & $(30.0)$ & 125 & (24.4) & $1.3(0.65,2.6)$ & 0.383 \\
\hline Goats or sheep & 13 & $(26.0)$ & 148 & (28.9) & $0.86(0.41,1.7)$ & 0.664 \\
\hline Pigs & 6 & (12.0) & 39 & (7.6) & $1.7(0.54,4.2)$ & 0.276 \\
\hline See livestock around house & 41 & (83.7) & 421 & (82.4) & $1.1(0.49,2.8)$ & 1.000 \\
\hline Cattle & 39 & $(79.6)$ & 342 & (66.8) & $4.1(1.6,9.8)$ & 0.067 \\
\hline Goats or sheep & 38 & (77.6) & 379 & (74.2) & $1.4(0.34,4.2)$ & 0.604 \\
\hline Pigs & 15 & (30.6) & 165 & (32.4) & $5.5(1.4,18.8)$ & 0.803 \\
\hline Slaughter livestock & 7 & (14.0) & 53 & (10.4) & $1.4(0.51,3.4)$ & 0.553 \\
\hline Cattle & 6 & (12.0) & 42 & $(8.2)$ & $1.5(0.50,3.9)$ & 0.492 \\
\hline Goats or sheep & 3 & $(6.0)$ & 21 & $(4.1)$ & $1.5(0.27,5.3)$ & 0.526 \\
\hline Pigs & 0 & $(0.0)$ & 5 & (1.0) & - & - \\
\hline \multicolumn{7}{|l|}{ Consume livestock products } \\
\hline Boiled or pasteurized milk & 37 & (74.0) & 397 & (77.7) & $0.82(0.41,1.7)$ & 0.660 \\
\hline Boiled or pasteurized dairy products & 2 & $(4.0)$ & 72 & (14.1) & $0.25(0.03,1.0)$ & 0.052 \\
\hline Raw milk & 0 & $(0.0)$ & 2 & $(0.4)$ & - & - \\
\hline Raw dairy products, total & 12 & (24.5) & 107 & (20.9) & $1.2(0.56,2.5)$ & 0.673 \\
\hline Cream & 0 & $(0.0)$ & 3 & $(0.6)$ & - & - \\
\hline Butter & 0 & $(0.0)$ & 5 & (1.0) & - & - \\
\hline Cheese & 0 & $(0.0)$ & 0 & $(0.0)$ & - & - \\
\hline Yogurt & 12 & (24.5) & 99 & (19.4) & $1.4(0.62,2.8)$ & 0.492 \\
\hline Other & 0 & $(0.0)$ & 1 & $(0.2)$ & - & - \\
\hline Raw livestock blood & 9 & (18.0) & 38 & (7.4) & $2.7(1.1,6.3)$ & 0.033 \\
\hline Cattle blood & 7 & (14.0) & 35 & (6.8) & $2.2(0.78,5.5)$ & 0.067 \\
\hline Goat or sheep blood & 3 & $(6.0)$ & 9 & (1.8) & $3.6(0.60,14.9)$ & 0.165 \\
\hline Pig blood & 1 & (2.0) & 3 & $(0.6)$ & $3.5(0.06,43.9)$ & 0.624 \\
\hline \multicolumn{7}{|l|}{ Exposure scales } \\
\hline Livestock contact, mean score (range) & $0.64(0,12.67)$ & $\mathrm{n} / \mathrm{a}$ & $0.64(0,11.54)$ & $\mathrm{n} / \mathrm{a}$ & $1.2(1.0,1.3)$ & 0.006 \\
\hline Cattle contact & $0.64(0,10.56)$ & $n / a$ & $0.64(0,11.26)$ & $n / a$ & $1.2(1.1,1.4)$ & 0.002 \\
\hline Goat or sheep contact & $0.64(0,10.28)$ & $\mathrm{n} / \mathrm{a}$ & $0.64(0,9.29)$ & $\mathrm{n} / \mathrm{a}$ & $1.2(1.0,1.4)$ & 0.019 \\
\hline Pig contact & $0.00(0,7.64)$ & $n / a$ & $0.00(0,9.11)$ & $n / a$ & $1.2(1.0,1.5)$ & 0.033 \\
\hline Livestock blood exposure & $0.00(0,10.28)$ & $n / a$ & $0.00(0,10.28)$ & $\mathrm{n} / \mathrm{a}$ & $1.1(1.0,1.2)$ & 0.037 \\
\hline Cattle blood & $0.00(0,10.28)$ & $n / a$ & $0.00(0,10.28)$ & $\mathrm{n} / \mathrm{a}$ & $1.1(0.99,1.2)$ & 0.086 \\
\hline Goat or sheep blood & $0.76(0,10.28)$ & $n / a$ & $0.36(0,10.28)$ & $n / a$ & $1.1(0.99,1.3)$ & 0.076 \\
\hline Pig blood & $0.23(0,4.26)$ & $\mathrm{n} / \mathrm{a}$ & $0.11(0,7.27)$ & $\mathrm{n} / \mathrm{a}$ & $1.2(0.87,1.57)$ & 0.298 \\
\hline
\end{tabular}

Cl= confidence interval; OR = odds ratio.

* Data not available for all participants for all variables; \% reflects the accurate denominator.

† Livestock - cattle, goats, pigs, and sheep.

$\ddagger$ Mean score (range), rather than $n(\%)$, is presented for all exposure scales. 
TABLE 4

Multivariable analysis of characteristics of participants with and without brucellosis, northern Tanzania, 2012-2014

\begin{tabular}{llll}
\hline \multicolumn{1}{c}{ Variable $^{\star}$} & \multicolumn{1}{c}{ OR } & \multicolumn{1}{c}{$(95 \% \mathrm{Cl})$} & $P$ value \\
\hline Assist sheep or goat births & 5.9 & $(1.4,25.2)$ & 0.015 \\
Age & 1.5 & $(1.1,2.0)$ & 0.007 \\
Cattle contact & 1.2 & $(1.0,1.4)$ & 0.016 \\
Consume boiled or pasteurized dairy & 0.12 & $(0.02,0.91)$ & 0.040 \\
$\quad$ products & & & \\
Residence outside Moshi Urban District & 0.57 & $(0.29,1.1)$ & 0.086 \\
Consume raw dairy products & 0.89 & $(0.43,1.9)$ & 0.764 \\
\hline
\end{tabular}

$\mathrm{Cl}=$ confidence interval; $\mathrm{OR}=$ odds ratio.

* Variables originally included-age, assist livestock births, assist cattle births, assist goat or sheep births, assist pig births, livestock blood contact, cattle blood contact, goat or sheep blood contact, pig blood contact, livestock contact, cattle contact, goat or sheep contact, pig contact, consume raw dairy, consume raw dain products, consume boiled or pasteurized dairy, consume boiled or pasteurized dairy products, district of residence.

While livestock-related occupations have been reported as risk factors for brucellosis in studies from sub-Saharan Africa, ${ }^{7,31}$ we did not observe an association between brucellosis and being a butcher, farmer, livestock attendant, or veterinarian. However, our data showed that activities involving livestock were not restricted to those who reported having livestock-related occupations. This highlights the importance of assessing specific behavioral risk factors rather than using proxies of risk, such as occupation or demographics.

We controlled for age in our multivariable model and observed that age was also an independent risk factor for brucellosis. We previously showed that increasing age was a risk factor for brucellosis in northern Tanzania, ${ }^{32}$ and older age has been identified as a risk factor for Brucella antibody seropositivity. ${ }^{16,33,34}$ These findings may be related to cumulative livestock exposure over time or to livestock-oriented activities that children do not perform.

While consumption of raw milk has been identified as a source of urban brucellosis in Uganda, ${ }^{35}$ we found no association between brucellosis and consumption of raw dairy or dairy products. One possible explanation is that the predominant livestock reservoir species in northern Tanzania is not the species from which people obtain most of their dairy. Interestingly, we observed a protective effect of boiled or pasteurized dairy product consumption. This could be due to the direct effect of better nutrition and health status in people who consume more boiled dairy products or reflect the effects of unobserved factors that are linked both with consumption of boiled dairy products and risk of brucellosis.

None of our 50 laboratory-confirmed brucellosis cases received a clinical diagnosis of brucellosis or effective brucellosis treatment during hospitalization. Several studies have shown limited healthcare provider awareness of zoonoses in Tanzania. ${ }^{36-38}$ Others have shown that despite the prevalence of endemic bacterial zoonoses such as brucellosis, clinicians overlook these diseases and over-diagnose malaria, ${ }^{15}$ as did the healthcare providers for our study participants. For every one laboratory-confirmed diagnosis of malaria, approximately 10 times that many participants were assigned a clinical diagnosis of malaria.

Our study had several limitations. We used self-reported district of residence in our analysis because of incomplete GPS data. Recall bias may have influenced participant responses about activities performed over the past month. The high ratio of independent variables to cases may have made our analysis underpowered to detect associations between brucellosis and individual behaviors. Most study participants were from urban and peri-urban zones, limiting our ability to assess brucellosis risk among rural dwellers. All brucellosis cases were diagnosed by serology rather than by culture, preventing analysis at the Brucella species level. Nearly onequarter of participants with Brucella antibodies had titers too high to be considered controls and too low to be considered cases. It was difficult to draw epidemiologic conclusions from those participants, as they may have been exposed to Brucella in the past, had active disease but failed to mount a substantial antibody responses, or tested positive for Brucella antibodies due to cross-reactions between antibodies to other gramnegative bacteria and Brucella test antigens. ${ }^{1}$ While the exclusion of such participants, along with participants missing convalescent titers, may have influenced our outcomes, an exploratory analysis showed no significant differences between included and excluded participants in terms of age, sex, tribe, household location, consumption of raw dairy, consumption of livestock blood, or birthing livestock. And finally, our selection of behaviors to include in the exposure scales may not have been sufficiently comprehensive or may have been too exclusive. While we acknowledge that there is scope to improve the development of such exposure scales and to validate them, we believe that grouping data using biologic plausibility, rather than purely statistical methods, offered several advantages. Most our participants engaged in multiple potentially risky behaviors, and our methods offered a way to tease out epidemiologically meaningful behavioral patterns. In addition, we were able to evaluate the risk of exposures to potentially infectious sources even though we were unable to directly measure those exposures in our questionnaire.

In summary, we identified risk factors for human brucellosis in northern Tanzania. Knowledge of these risk factors may contribute to disease prevention and control efforts and may assist clinicians with risk stratification. Our research could be extended in a number of ways. To help target provision of education and personal protective equipment in northern Tanzania, levels of exposure to potentially infectious livestock body fluids could be quantified through bioaerosol sampling, detailed observation of livestock-related activities, and indepth interviews. To develop livestock brucellosis vaccination strategies for northern Tanzania, bacterial isolates from human and livestock cases are needed to identify infecting Brucella species. Because pastoralists are more likely to have higher levels of exposure to livestock than nonpastoralists, it would be useful to repeat our study in a pastoralist context. In the meantime, the use of personal protective equipment among those with high levels of livestock contact, especially during the livestock birthing process, may help reduce disease transmission. Education efforts to promote boiling of milk and dairy products sold in urban areas may also help prevent disease. Finally, improving clinician awareness that not all fevers are malaria and strengthening diagnostic services for nonmalaria fever would improve the recognition and appropriate management of patients with brucellosis.

Received February 17, 2017. Accepted for publication October 8, 2017.

Published online December 11, 2017.

Acknowledgments: We thank Francis P. Karia for providing administrative support; Michael E. Butoyi, Mariam Barabara, Godfrey S. Mushi, Anna Mwalla, Lillian E. Ngowi, Flora M. Nkya, and Winfrida H. Shirima for 
reviewing and enrolling study participants; Luig Mbuya, Yusuph Msuya, Leila Sawe, Tumsifu G. Tarimo, and Aaron E. Tesha for risk factor questionnaire administration and research assistance; Euphrasia Mariki and Edward Singo for managing participant followup; Isaac A. Afwamba, Augustine M. Musyoka Philoteus, Sakasaka, Remigi P. Swai, and Thomas Walongo for laboratory assistance; and Robert Chuwa, Enock Kessy, and Evaline Ndosi for their assistance in data entry. We are grateful to William A. de Glanville for his contributions to the manuscript. We acknowledge the Hubert-Yeargan Center for Global Health at Duke University for critical infrastructure support for the Kilimanjaro Christian Medical Centre-Duke University Collaboration. We are grateful to the leadership, clinicians, and patients of $\mathrm{KCMC}$ and $\mathrm{MRRH}$ for their contributions to this research.

Financial support: This research was supported by the joint US National Institutes of Health (NIH, www.nih.gov)-National Science Foundation (NSF, www.nsf.gov) Ecology of Infectious Disease program (R01TW009237), and the Zoonoses and Emerging Livestock Systems initiative (www.bbsrc.ac.uk/research/international/zels/) funded through RCUK, DFID, DSTL (Biotechnology and Biological Sciences Research Council grant numbers BB/J010367/1, BB/ L018926, BB/L017679, BB/L018845), and in part by an US NIH International Studies on AIDS Associated Co-infections (ISAAC) award (U01 Al062563) and in part by the Bill \& Melinda Gates Foundation funded Typhoid Fever Surveillance in sub-Saharan Africa Program (TSAP) grant (OPPGH5231). Authors received support from a US NIH Research Training Grant funded by the Fogarty International Center and the National Institute of Mental Health R25 TW009337 (S. C.-G., M. P. R.), a University of Otago Frances G. Cotter Scholarship (M. J. M.), and US NIH grants NIAID K23 Al116869 (M. P. R.) and NIAID-AI007392 (H. M. B.). The funders had no role in study design, data collection and analysis, decision to publish, or preparation of the manuscript.

Disclosure: Presented in part at the American Society of Tropical Medicine and Hygiene 65th Annual Meeting, Atlanta, GA, November 13-17, 2016, Abstract 1773.

Disclaimer: The findings and conclusions in this report are those of the authors and do not necessarily represent the official position of the Centers for Disease Control and Prevention. Use of trade names and commercial sources is for identification only and does not imply endorsement by the US Department of Health and Human Services or the Centers for Disease Control and Prevention.

Authors' addresses: Shama Cash-Goldwasser, Duke Global Health Institute, Duke University, Durham, NC, and Kilimanjaro Christian Medical Centre, KCMC-Duke University Collaboration, Moshi, Kilimanjaro, Tanzania, E-mail: shamacg@gmail.com. Michael J. Maze, Centre for International Health, University of Otago, Dunedin, New Zealand, and Kilimanjaro Christian Medical Centre, KCMC-Duke University Collaboration, Moshi, Kilimanjaro, Tanzania, E-mail: mazmi604@student.otago.ac.nz. Matthew P. Rubach, Kilimanjaro Christian Medical Centre, KCMC-Duke Collaboration, Moshi, Kilimanjaro, Tanzania, and Division of Infectious Diseases, Duke University Medical Center, Durham, NC, E-mail: matthew.rubach@duke. edu. Holly M. Biggs, Division of Infectious Diseases, Duke University Medical Center, Durham, NC, E-mail: hollybiggs1@gmail.com. Robyn A. Stoddard, Bacterial Special Pathogens Branch, Centers for Disease Control and Prevention, Atlanta, GA, E-mail: frd8@cdc.gov. Katrina J. Sharples, Department of Mathematics and Statistics and Department of Medicine, University of Otago, Dunedin, New Zealand, E-mail: katrina.sharples@otago.ac.nz. Jo E. B. Halliday and Sarah Cleaveland, College of Medical, Veterinary and Life Sciences, University of Glasgow, Glasgow, United Kingdiom, E-mails: jo.halliday@ glasgow.ac.uk and sarah.cleaveland@glasgow.ac.uk. Michael C. Shand, Boyd Orr Centre for Population and Ecosystem Health, Institute of Biodiversity, Animal Health and Comparative Medicine, Glasgow, United Kingdom, E-mail: mike.shand@glasgow.ac.uk. Blandina T. Mmbaga, Kilimanjaro Christian Medical Center, KCMCDuke University Collaboration, Moshi, Tanzania, Duke Global Health Institute, Duke University Medical Center, Durham, NC, and Kilimanjaro Christian Medical University College, KCMC-Duke University Collaboration, Moshi, Kilimanjaro, Tanzania, E-mail: blaymt@yahoo. com. Charles Muiruri, Duke Global Health Institute, Duke University, Durham, NC, E-mail: muiru001@duke.edu. Wilbrod Saganda and Bingileki F. Lwezaula, Department of Medicine, Mawenzi Regional
Referral Hospital, Moshi, Tanzania, E-mails: wilbrodsaganda@gmail. com and Iwezaula@gmail.com. Rudovick R. Kazwala, Department of Veterinary Medicine and Public Health, Sokoine University of Agriculture, Morogoro, Tanzania, E-mail: kazwala@gmail.com. Venance P. Maro, Department of Medicine, Kilimanjaro Christian Medical Centre, Moshi, Kilimanjaro, Tanzania, and Department of Medicine, Kilimanjaro Christian Medical University College, Moshi, Kilimanjaro, Tanzania, E-mail: venmaro@ymail.com. John A. Crump, Duke Global Health Institute, Duke University, Durham, NC, Centre for International Health, University of Otago, Dunedin, New Zealand, Division of Infectious Diseases, Duke University Medical Center, Durham, NC, and Department of Medicine, Kilimanjaro Christian Medical University College, Moshi, Kilimanjaro, Tanzania, E-mail: john.crump@otago.ac.nz.

This is an open-access article distributed under the terms of the Creative Commons Attribution License, which permits unrestricted use, distribution, and reproduction in any medium, provided the original author and source are credited.

\section{REFERENCES}

1. Corbel MJ, 2006. Brucellosis in Humans and Animals. Geneva, Switzerland: World Health Organization and Food and Agriculture Organization of the United Nations.

2. Pappas G, Papadimitriou P, Akritidis N, Christou L, Tsianos EV, 2006. The new global map of human brucellosis. Lancet Infect Dis 6: 91-99.

3. Crump JA et al., 2011. Invasive bacterial and fungal infections among hospitalized HIV-infected and HIV-uninfected adults and adolescents in northern Tanzania. Clin Infect Dis 52: 341-348.

4. Reddy EA, Shaw AV, Crump JA, 2010. Community-acquired bloodstream infections in Africa: a systematic review and metaanalysis. Lancet Infect Dis 10: 417-432.

5. Rubach MP, Halliday JE, Cleaveland S, Crump JA, 2013. Brucellosis in low-income and middle-income countries. Curr Opin Infect Dis 26: 404-412.

6. Corbel MJ, 1997. Brucellosis: an overview. Emerg Infect Dis 3: 213-221.

7. McDermott J, Grace D, Zinsstag J, 2013. Economics of brucellosis impact and control in low-income countries. Rev Sci Tech 32: 249-261.

8. Godfroid J, Cloeckaert A, Liautard JP, Kohler S, Fretin D, Walravens K, Garin-Bastuji B, Letesson JJ, 2005. From the discovery of the Malta fever's agent to the discovery of a marine mammal reservoir, brucellosis has continuously been a reemerging zoonosis. Vet Res 36: 313-326.

9. McDermott JJ, Arimi SM, 2002. Brucellosis in sub-Saharan Africa: epidemiology, control and impact. Vet Microbiol 90: 111-134.

10. Godfroid J, Nielsen K, Saegerman C, 2010. Diagnosis of brucellosis in livestock and wildlife. Croat Med J 51:296-305.

11. Pappas G, Akritidis N, Bosilkovski M, Tsianos E, 2005. Brucellosis. N Engl J Med 352: 2325-2336.

12. Shimol SB, Dukhan L, Belmaker I, Bardenstein S, Sibirsky D, Barrett C, Greenberg D, 2012. Human brucellosis outbreak acquired through camel milk ingestion in southern Israel. Isr Med Assoc J 14: 475-478.

13. Chomel BB, DeBess EE, Mangiamele DM, Reilly KF, Farver TB, Sun RK, Barrett LR, 1994. Changing trends in the epidemiology of human brucellosis in California from 1973 to 1992: a shift toward foodborne transmission. J Infect Dis 170: 1216-1223.

14. Schelling E, Diguimbaye C, Daoud S, Nicolet J, Boerlin P, Tanner M, Zinsstag J, 2003. Brucellosis and Q-fever seroprevalences of nomadic pastoralists and their livestock in Chad. Prev Vet Med 61: 279-293.

15. Crump JA et al., 2013. Etiology of severe non-malaria febrile illness in northern Tanzania: a prospective cohort study. PLOS Negl Trop Dis 7: e2324.

16. Swai ES, Schoonman L, 2009. Human brucellosis: seroprevalence and risk factors related to high risk occupational groups in Tanga Municipality, Tanzania. Zoonoses Public Health 56: 183-187.

17. John K, Fitzpatrick J, French N, Kazwala R, Kambarage D, Mfinanga G, MacMillan A, Cleaveland S, 2010. Quantifying risk factors for human brucellosis in rural northern Tanzania. PLOS One 5: e9968. 
18. Shirima GM, Masola SN, Malangu ON, Schumaker BA, 2014. Outbreak investigation and control case report of brucellosis: experience from livestock research centre, Mpwapwa, Tanzania. Onderstepoort J Vet Res 81.

19. Government of Tanzania, 2002. Population and Housing Census. Dar es Salaam, Tanzania: National Bureau of Statistics, Government of Tanzania.

20. Food and Agriculture Organization of the United Nations, Livestock Information, Sector Analysis and Policy Branch, 2005. Livestock Sector Brief. Tanzania: United Republic of Tanzania.

21. Crump JA et al., 2011. Invasive bacterial and fungal infections among hospitalized HIV-infected and HIV-uninfected children and infants in northern Tanzania. Trop Med Int Health 16: 830-837.

22. Brown SL, Klein GC, McKinney FT, Jones WL, 1981. Safranin O-stained antigen microagglutination test for detection of Brucella antibodies. J Clin Microbiol 13: 398-400.

23. CDC, 2010. Brucellosis Case Definition. Available at: https:// wwwn.cdc.gov/nndss/conditions/brucellosis/case-definition/ 2010/. Accessed August 1, 2015.

24. Vyas S, Kumaranayake L, 2006. Constructing socio-economic status indices: how to use principal components analysis. Health Policy Plan 21: 459-468.

25 . Saaty $T, 2008$. Decision making with the analytic hierarchy process. Int J Serv Sci 1: 83-98.

26. Saaty $T, 1990$. How to make a decision: the analytic hierarchy process. Eur J Oper Res 48: 9-26.

27. Orsel K, Ho J, Hatfield J, Manyama M, Ribble C, van der Meer F, 2015. Brucellosis serology as an alternative diagnostic test for patients with malaria-like symptoms. Tanzan J Health Res 17.

28. Viana M, Shirima GM, John KS, Fitzpatrick J, Kazwala RR, Buza JJ, Cleaveland S, Haydon DT, Halliday JE, 2016. Integrating serological and genetic data to quantify cross-species transmission: brucellosis as a case study. Parasitology 143: 821-834.

29. Asiimwe BB, Kansiime C, Rwego IB, 2015. Risk factors for human brucellosis in agro-pastoralist communities of south western Uganda: a case-control study. BMC Res Notes 8: 405.
30. Dean AS, Bonfoh B, Kulo AE, Boukaya GA, Amidou M, Hattendorf J, Pilo P, Schelling E, 2013. Epidemiology of brucellosis and Q FEVER in linked human and animal populations in northern Togo. PLoS One 8: e71501.

31. Ducrotoy M, Bertu WJ, Matope G, Cadmus S, Conde-Álvarez R, Gusi AM, Welburn S, Ocholi R, Blasco JM, Moriyón I, 2015. Brucellosis in Sub-Saharan Africa: current challenges for management, diagnosis and control. Acta Trop 165: 179-193.

32. Bouley AJ et al., 2012. Brucellosis among hospitalized febrile patients in northern Tanzania. Am J Trop Med Hyg 87: 1105-1111.

33. Cetinkaya Z, Aktepe OC, Ciftci IH, Demirel R, 2005. Seroprevalence of human brucellosis in a rural area of western Anatolia, Turkey. J Health Popul Nutr 23: 137-141.

34. Osoro EM et al., 2015. Strong association between human and animal Brucella seropositivity in a linked study in Kenya, 2012-2013. Am J Trop Med Hyg 93: 224-231.

35. Makita K, Fèvre EM, Waiswa $\mathrm{C}$, Kaboyo W, Bronsvoort BMdeC, Eisler MC, Welburn SC, 2008. Human brucellosis in urban and peri-urban areas of Kampala, Uganda. Ann N Y Acad Sci 1149: 309-311.

36. Chipwaza B, Mugasa JP, Mayumana I, Amuri M, Makungu C, Gwakisa PS, 2014. Community knowledge and attitudes and health workers' practices regarding non-malaria febrile illnesses in eastern Tanzania. PLoS Negl Trop Dis 8: e2896.

37. John K, Kazwala R, Mfinanga GS, 2008. Knowledge of causes, clinical features and diagnosis of common zoonoses among medical practitioners in Tanzania. BMC Infect Dis 8: 162.

38. Zhang HL, Mnzava KW, Mitchell ST, Melubo ML, Kibona TJ, Cleaveland S, Kazwala RR, Crump JA, Sharp JP, Halliday $\mathrm{JE}, 2016$. Mixed methods survey of zoonotic disease awareness and practice among animal and human healthcare providers in Moshi, Tanzania. PLoS Negl Trop Dis 10: e0004476. 